\title{
Effect of Lipid Molecules on Twisting Motions of DNA Helix Studied by Fluorescence Polarization Anisotropy
}

\author{
Hideki Kimura, Shinjiro Machida, ${ }^{\dagger}$ Kazuyuki Horie \\ and Yoshio OKaHATA* \\ Department of Chemistry and Biotechnology, Graduate School of Engineering, The University of Tokyo, \\ 7-3-1 Hongo, Bunkyo-ku, Tokyo 113-8656, Japan \\ * Department of Biomolecular Engineering, Tokyo Institute of Technology, \\ Nagatsuta, Midori-ku, Yokohama 226-8501, Japan
}

(Received February 6, 1998)

\begin{abstract}
Time-resolved fluorescence polarization anisotropy (FPA) of two dyes (acridine orange and ethidium bromide) intercalated in a raw DNA and a novel DNA-lipid complex has been measured on nanosecond timescale to evaluate the effect of lipid molecules on the twisting motions of DNA helix. Steady-state fluorescence spectra show that intercalated dyes are not influenced much by solvents compared with free dyes, suggesting the similar microscopic environments of intercalated chromophores. The FPA decay times of both dyes are about twice larger in the DNA-lipid complex than in the raw DNA, which indicates that the lipid molecules around the helix make the twisting motion slower. The FPA decay profiles fit very well with double-exponential functions having time constants differing much with each other, suggesting that there exists slow and quick elements in the twisting motions.

KEY WORDS DNA-Lipid Complex / Intercalation / Acridine Orange / Ethidium Bromide / Time-Resolved Fluorescence Anisotropy / Twisting Motion /
\end{abstract}

It is quite well-known that DNA is an important source of biological information depending on base sequences. In a living cell, the information stored as the base sequence along the DNA chain is expressed as the production of a rich variety of proteins in a self-regulatory manner. Thus, the interaction of DNA with various kinds of chemical components in a cell such as inorganic ions, proteins, and cationic lipids is essentially important for the regulation of living state. ${ }^{1}$

In these years the dynamical behavior of DNA has also attracted interest, since in order to understand processes such as intracellular DNA rearrangements or DNA-protein interactions, it is very important to possess a detailed knowledge about the structure and especially about internal motions of DNAs. ${ }^{2}$ Though the conformational flexibility and dynamics of the DNA helix are very complex, there are mainly two kinds of internal motions of DNA, i.e., bending and twisting. The bending motions occur on the timescale of microseconds and the twisting proceeds in nanosecond range. The balance of these two motions plays a fundamental role in the biological activity of DNA in many instances.

Dynamical aspects of DNA have been investigated by monitoring the elastic properties of the double helix. Among the techniques, time-resolved fluorescence polarization anisotropy (FPA) measurement is particularly suitable for detecting the twisting motions. FPA contains information on the rotational Brownian motions of macromolecules bearing fluorescence probes either intrinsically or extrinsically. We can regard DNA as a macromolecule which consists of a linear sequence of linked rodlike segments, each of which has cylindrical symmetry. Thus, the time-resolved FPA of the dye molecules intercalated between adjacent base-pairs of DNA on nanosecond timescale can be a sensitive measure of the twisting motions of DNA. ${ }^{3,4}$

\footnotetext{
$\dagger$ To whom correspondence should be addressed.
}

Recently, a novel DNA species, DNA-lipid complex, that has a lot of unique characteristics, has been constructed. ${ }^{5}$ The structure of the DNA-lipid complex is shown in Figure 1. This complex is prepared by such a simple process as replacing counter cations by cationic amphiphilic lipids. Chain-like lipid molecules attach to phosphate anions around the helix with the ratio of $1: 1$. Being surrounded by amphiphilic molecules, this complex is soluble in most organic media. In organic media, the double helical structures are retained and intercalation behavior of dye molecules is exhibited. ${ }^{6}$ This DNA-lipid complex can form a transparent film with solvent casting from organic solution, and the drawing of the film makes it possible to align the DNA chains. Therefore, the DNA-lipid complex has a possibility of being used for various kinds of applications such as an adsorber of cancer suspect agents and a light-polarizing filter. ${ }^{7}$

In this paper, we focus on this peculiar structure of the DNA-lipid complex and adopt it for the study of internal motion, especially twisting, of the DNA helix. The remarkable structure, constructed by long, bold and rather flexible cylinder surrounded with thin and hairy chains, induces us to consider the interaction of DNA helix with the chain-like lipid molecules bound around it. Thanks to the intercalation behavior of dye molecules into the DNA-lipid complex, the twisting motion of this complex in organic solution can be monitored by the time-resolved FPA measurement of the intercalated dyes on nanosecond timescale as mentioned above. In order to evaluate the effect of lipid molecules around the helix on the twisting motion, we compared the decay times of time-resolved FPA for a raw DNA and the DNA-lipid complex, by using acridine orange (AO) and ethidium bromide (EB) as a probe dye. From absorption spectra and circular dichroism (CD) spectra, intercalation of dyes are confirmed. Fluorescence spectra, steady-state FPA, and fluorescence lifetime in addition to the timeresolved FPA are measured to get information about 

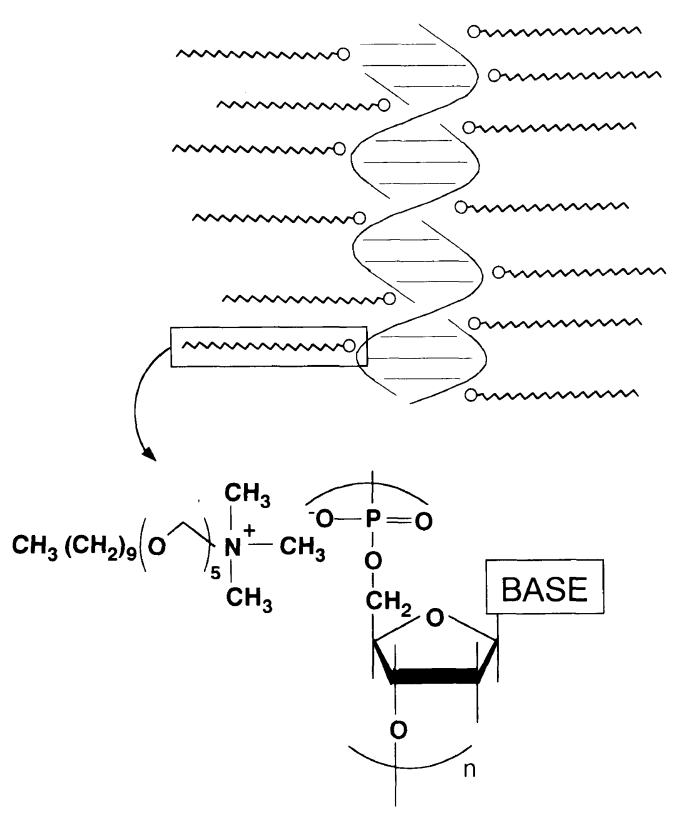

Figure 1. Schematic illustration of DNA-lipid complex.

spectroscopic properties of the dyes intercalated in the DNA-lipid complex.

\section{EXPERIMENTAL}

AO and EB were obtained from Sigma Chemical Co. (St. Louis). As for a raw DNA, sodium salts of DNA from Salmon testes $\left(c a .2000 \mathrm{bp}\right.$, average $M_{w}: 1.3 \times 10^{6}$ ) were purchased from Sigma Chem. Co. The DNA-lipid complex was prepared by the method reported previously, ${ }^{7}$ whose DNA-parts consist of the same ones as we employed as the raw DNA. Standard phosphate buffer ( $\mathrm{pH}$ 6.9) was used for the solvent for the raw DNA samples, and the DNA-lipid complex samples were dissolved in $\mathrm{CHCl}_{3} / \mathrm{MeOH} / \mathrm{H}_{2} \mathrm{O}(4: 1: 0.05)$ mixture. The concentration of $\mathrm{AO}$ is $1 \times 10^{-5} \mathrm{~mol} \mathrm{~L}^{-1}$ and the concentration of EB is $2 \times 10^{-5} \mathrm{~mol} \mathrm{~L}^{-1}$, both for raw DNA and DNA-lipid complex samples. All these reagents were used without further purification.

Intercalative conditions were prepared at phosphate/ dye $(\mathrm{P} / \mathrm{D})$ ratio of 100 and 50 for $\mathrm{AO}$ and $\mathrm{EB}$, respectively. Samples were put into a $1 \mathrm{~cm}$ thick quartz cell for all measurements. Viscosities of the phosphate buffer and the $\mathrm{CHCl}_{3} / \mathrm{MeOH} / \mathrm{H}_{2} \mathrm{O}(4: 1: 0.05)$ mixture were measured using Ostwald-type viscometer. CD spectra of the dyes which are free or with the raw DNA in buffer, and free or with the DNA-lipid complex in $\mathrm{CHCl} / 3 / \mathrm{MeOH} / \mathrm{H}_{2} \mathrm{O}$ solution were taken with a $\mathrm{J}-500$ spectropolarimeter (JASCO, Tokyo). Fluorescence spectra and steady-state FPA measurements were carried out with a Hitachi 850 fluorescence spectrophotometer. The solutions were excited in a front-face arrangement to minimize self-absorption. The values of steady-state FPA, $r$, were obtained from eq 1 including the $g$ factor,

$$
r=\left(i_{\|}-g i_{\perp}\right) /\left(i_{\|}+2 g i_{\perp}\right)
$$

where $i_{\|}$and $i_{\perp}$ are the parallel and perpendicular components, respectively, of the observed fluorescence intensity to the polarization of the exciting light. Fluorescence lifetime and time-resolved FPA measure- ments were performed using a single-photon-counting apparatus (C4780, Hamamatsu Photonics, Hamamatsu). A $\mathrm{N}_{2}$-laser (LN120, Laser Photonics, Orlando)-pumped coumarin-503 dye laser (LN120C2) was used for generating 500-nm light for exciting the region of the dye's absorption spectra. The fluorescence decays were obtained with a Hamamatsu streak scope (C4334). The $g$ factor for the time-resolved FPA measurement was regarded to be 1.00 because we used an optical fiber for guiding the fluorescence to the streak scope, which made a linearly-polarized light to randomly-polarized light.

\section{RESULTS AND DISCUSSION}

The $\mathrm{AO}$ and $\mathrm{EB}$ have their absorption maxima at $\sim 500 \mathrm{~nm}$ both in buffer and $\mathrm{CHCl}_{3} / \mathrm{MeOH} / \mathrm{H}_{2} \mathrm{O}$ solutions. The absorption maxima do not change so much in the presence of the raw DNA or the DNA-lipid complex. In the $\mathrm{CD}$ spectra of $\mathrm{AO}$, positive Cotton effect is observed at around $500 \mathrm{~nm}$ in the presence of DNA. With respect to $\mathrm{EB}$, the addition of DNA induces negative cotton effect around $500 \mathrm{~nm}$. Thus, we regard that $\mathrm{AO}$ and $\mathrm{EB}$ intercalate in the raw DNA or the DNA-lipid complex in buffer or $\mathrm{CHCl}_{3} / \mathrm{MeOH} / \mathrm{H}_{2} \mathrm{O}$ solutions.

Figure 2 shows fluorescence and its excitation spectra of free (a) and intercalating (b) AO in buffer (solid lines) and $\mathrm{CHCl}_{3} / \mathrm{MeOH} / \mathrm{H}_{2} \mathrm{O}$ (dotted lines) solutions. As for free AO (Figure 2(a)), excitation peaks are observed around $490 \mathrm{~nm}$ both in the buffer and the $\mathrm{CHCl}_{3} /$ $\mathrm{MeOH} / \mathrm{H}_{2} \mathrm{O}$ solution, but the $\mathrm{CHCl}_{3} / \mathrm{MeOH} / \mathrm{H}_{2} \mathrm{O}$ solution has its excitation peak at a little shorter wavelength. The emission peak wavelength of free $\mathrm{AO}$ in the $\mathrm{CHCl}_{3} / \mathrm{MeOH} / \mathrm{H}_{2} \mathrm{O}$ solution is also shorter than that in the buffer solution $\left(515 \mathrm{~nm}\right.$ in the $\mathrm{CHCl}_{3} / \mathrm{MeOH} /$ $\mathrm{H}_{2} \mathrm{O}$ solution and $530 \mathrm{~nm}$ in the buffer solution). When the AO is intercalating in the raw DNA or the DNA-lipid complex (Figure 2(b)), a little shift to shorter wavelength of the emission and excitation peaks are observed with changing solvents from the buffer to the $\mathrm{CHCl}_{3} / \mathrm{MeOH} /$ $\mathrm{H}_{2} \mathrm{O}$ solution as is observed for the free AO. However, the shift of the emission peak of the intercalating $\mathrm{AO}$ is not so large as that of the free one. In Figure 2(a) the fluorescence intensity of the free AO strikingly increases with changing solvents from the buffer to the $\mathrm{CHCl}_{3}$ / $\mathrm{MeOH} / \mathrm{H}_{2} \mathrm{O}$ solution. On the other hand, in Figure 2(b) the fluorescence intensity does not increase but slightly decreases with changing solvents from the buffer to the $\mathrm{CHCl}_{3} / \mathrm{MeOH} / \mathrm{H}_{2} \mathrm{O}$ solution in the intercalative conditions. The solvents have a strong influence on the fluorescence properties of the free $\mathrm{AO}$, but do not influence on those of the intercalating one. It is reasonable because the intercalating dye molecules are sandwiched by the base pairs of the DNA helix and their fluorescence properties are not likely to be influenced by the solvent outside.

As for EB, the fluorescence behavior of the free and the intercalating dye in the buffer and the $\mathrm{CHCl}_{3} /$ $\mathrm{MeOH} / \mathrm{H}_{2} \mathrm{O}$ solutions is similar to that of $\mathrm{AO}$. For free $\mathrm{EB}$, the fluorescence intensity is much smaller in the buffer solution than that in the $\mathrm{CHCl}_{3} / \mathrm{MeOH} / \mathrm{H}_{2} \mathrm{O}$ solution, and the excitation and emission peak wavelengths are longer in the buffer solution than those 

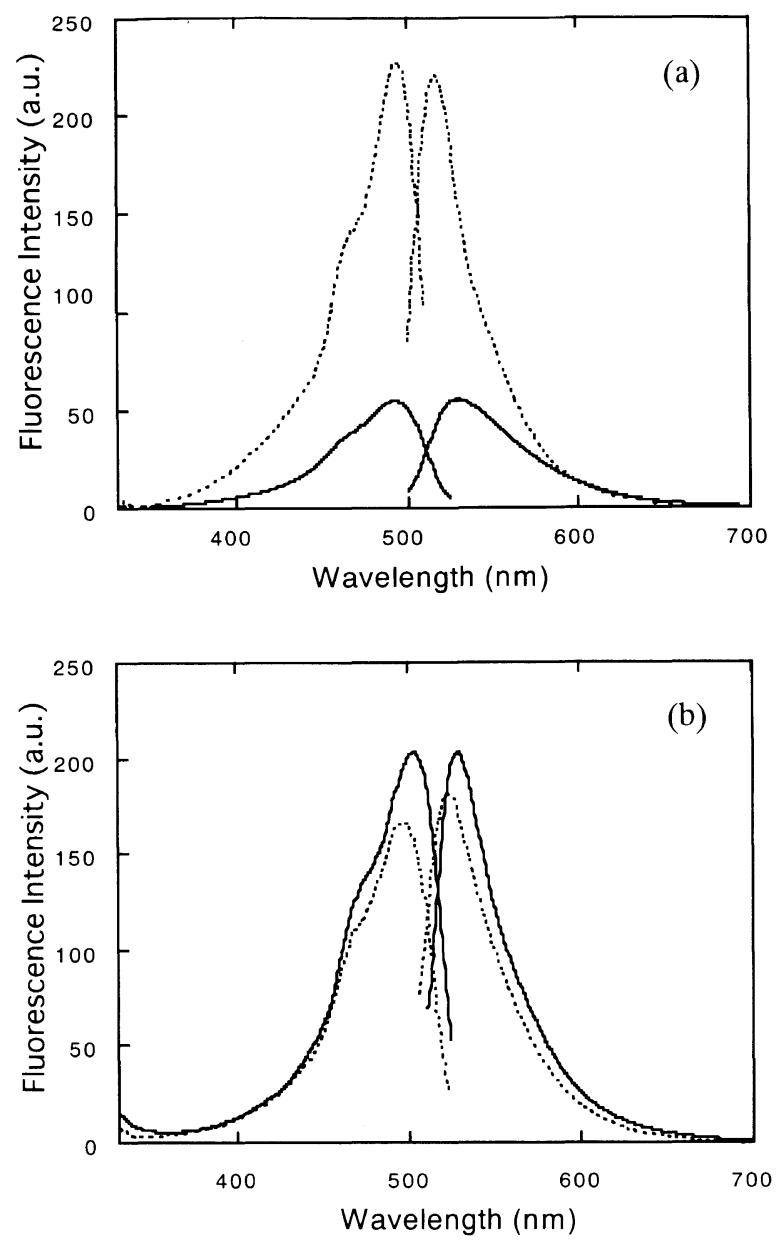

Figure 2. Fluorescence spectra of $\mathrm{AO}$ in buffer and $\mathrm{CHCl}_{3} / \mathrm{MeOH}$ $\mathrm{H}_{2} \mathrm{O}(4: 1: 0.05)$ solutions at the concentration of $1 \times 10^{-5} \mathrm{~mol} \mathrm{~L}^{-1}$. (a), free in buffer (solid lines) and $\mathrm{CHCl}_{3} / \mathrm{MeOH} / \mathrm{H}_{2} \mathrm{O}$ (dotted lines) solution (rightside, emission spectra excited at $490 \mathrm{~nm}$; leftside, excitation spectra monitored at $530 \mathrm{~nm}$ for buffer and $515 \mathrm{~nm}$ for $\mathrm{CHCl}_{3} / \mathrm{MeOH} / \mathrm{H}_{2} \mathrm{O}$ ); (b), intercalating in the raw DNA in buffer (solid lines) and in the DNA-lipid complex in $\mathrm{CHCl}_{3} / \mathrm{MeOH} / \mathrm{H}_{2} \mathrm{O}$ (dotted lines) (rightside, emission spectra excited at $500 \mathrm{~nm}$; leftside, excitation spectra monitored at $530 \mathrm{~nm}$ ).

in the $\mathrm{CHCl}_{3} / \mathrm{MeOH} / \mathrm{H}_{2} \mathrm{O}$ solution, as observed for the free AO. When EB is intercalating in the raw DNA or the DNA-lipid complex, the intensity and the peak wavelengths of the buffer and $\mathrm{CHCl}_{3} / \mathrm{MeOH} / \mathrm{H}_{2} \mathrm{O}$ solutions become similar. Therefore, we can conclude that the microscopic environments of the intercalating dye molecules are similar in the raw DNA and the DNA-lipid complex. Although the DNA phosphate has different counter cations, namely, $\mathrm{Na}^{+}$for the raw DNA and a cationic lipid for the DNA-lipid complex, the fluorescence properties are not so much affected by the counter cations.

Table I shows steady-state FPA and fluorescence lifetime of the dyes in buffer with and without the raw DNA, and in the $\mathrm{CHCl}_{3} / \mathrm{MeOH} / \mathrm{H}_{2} \mathrm{O}$ solution with and without the DNA-lipid complex. The fluorescence decay profiles for the dyes intercalating to the raw DNA or to the DNA-lipid complex were obtained as $i_{\|}(t)+2 i_{\perp}(t)$, where $i_{\|}(t)$ and $i_{\perp}(t)$ represent the parallel and perpendicular components, respectively, of the time-dependent fluorescence intensity to the polarization of the exciting light. Figure 3 shows the fluorescence decay
Table I. Steady-state FPA and fluorescence lifetime of AO and $\mathrm{EB}$, in buffer solution with and without raw DNA, and in $\mathrm{CHCl}_{3} / \mathrm{MeOH} / \mathrm{H}_{2} \mathrm{O}(4: 1: 0.05)$ solution with and without the DNA-lipid complex. The $\mathrm{P} / \mathrm{D}$ ratio is 100 for $\mathrm{AO}$ and 50 for $\mathrm{EB}$.

\begin{tabular}{|c|c|c|c|c|}
\hline \multirow{2}{*}{ Dye conditions } & \multicolumn{2}{|c|}{ Steady-state FPA } & \multicolumn{2}{|c|}{ Lifetime/ns } \\
\hline & $\mathrm{AO}$ & EB & $\mathrm{AO}$ & EB \\
\hline Free in buffer & 0.025 & 0.025 & 1.6 & 2.2 \\
\hline In buffer with the raw DNA & 0.134 & 0.096 & 7.1 & 27.8 \\
\hline Free in $\mathrm{CHCl}_{3} / \mathrm{MeOH} / \mathrm{H}_{2} \mathrm{O}$ & 0.011 & 0.006 & 3.2 & 6.4 \\
\hline $\begin{array}{l}\text { In } \mathrm{CHCl}_{3} / \mathrm{MeOH} / \mathrm{H}_{2} \mathrm{O} \text { with } \\
\text { the DNA-lipid complex }\end{array}$ & 0.095 & 0.133 & 4.3 & 13.6 \\
\hline
\end{tabular}

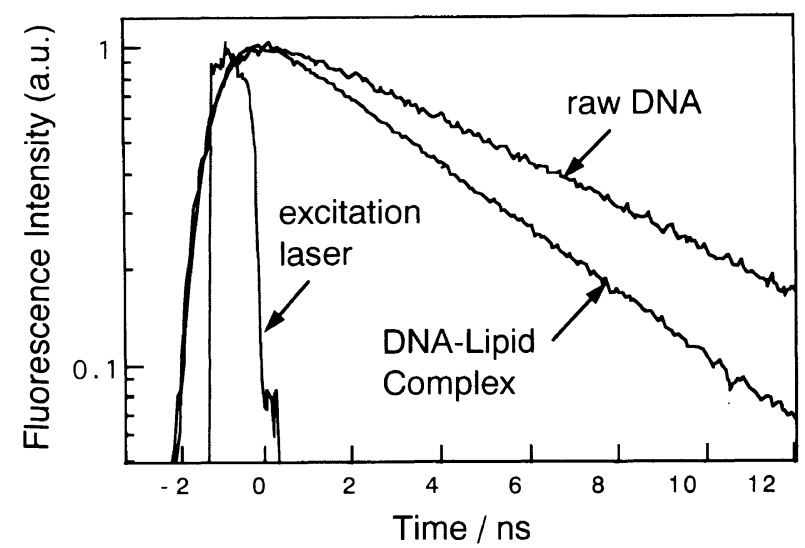

Figure 3. Fluorescence emission decay profiles, $i_{\| \mid}(t)+2 i_{\perp}(t)$, of $\mathrm{AO}$ intercalated in the raw DNA and the DNA-lipid complex. The profile of excitation laser pulse is also shown.

profile of $\mathrm{AO}$ in buffer with the raw DNA, and in $\mathrm{CHCl}_{3} / \mathrm{MeOH} / \mathrm{H}_{2} \mathrm{O}$ with the DNA-lipid complex. The profile of the excitation laser is also shown in Figure 3. The fluorescence lifetimes for the dyes with the raw DNA or the DNA-lipid complex were obtained without the deconvolution procedure. The fluorescence decay profiles for free dyes were measured without a polarization filter and deconvoluted by the profile of the excitation laser. The lifetimes were obtained by fitting the decays with a single-exponential function. In Table I the FPA values are very small in free solutions and are large in the intercalating state due to the restriction of the motion by the DNA helix. In the buffer solution, the fluorescence lifetimes of these dyes are known to be significantly longer in the intercalating state. In the $\mathrm{CHCl}_{3} / \mathrm{MeOH} / \mathrm{H}_{2} \mathrm{O}$ solution with the DNA-lipid complex, the fluorescence lifetimes are longer in the intercalating state than those on free condition as the case of the buffer solution. But the difference in lifetime between the intercalating and free states for the case of the DNA-lipid complex is much smaller than that for the case of the raw DNA. This result is reflected by the similar fluorescence intensities of the dyes between the free and intercalating state in the $\mathrm{CHCl}_{3} / \mathrm{MeOH} / \mathrm{H}_{2} \mathrm{O}$ solution compared to that in the buffer solution (see Figure 2).

Time-dependent FPA, $r(t)$, is calculated from $i_{\|}(t)$ and $i_{\perp}(t)$ according to eq $2 .^{8}$

$$
r(t)=\left[i_{\|}(t)-i_{\perp}(t)\right] /\left[i_{\|}(t)+2 i_{\perp}(t)\right]
$$

The anisotropy decays, $r(t)$, of the dyes intercalating 

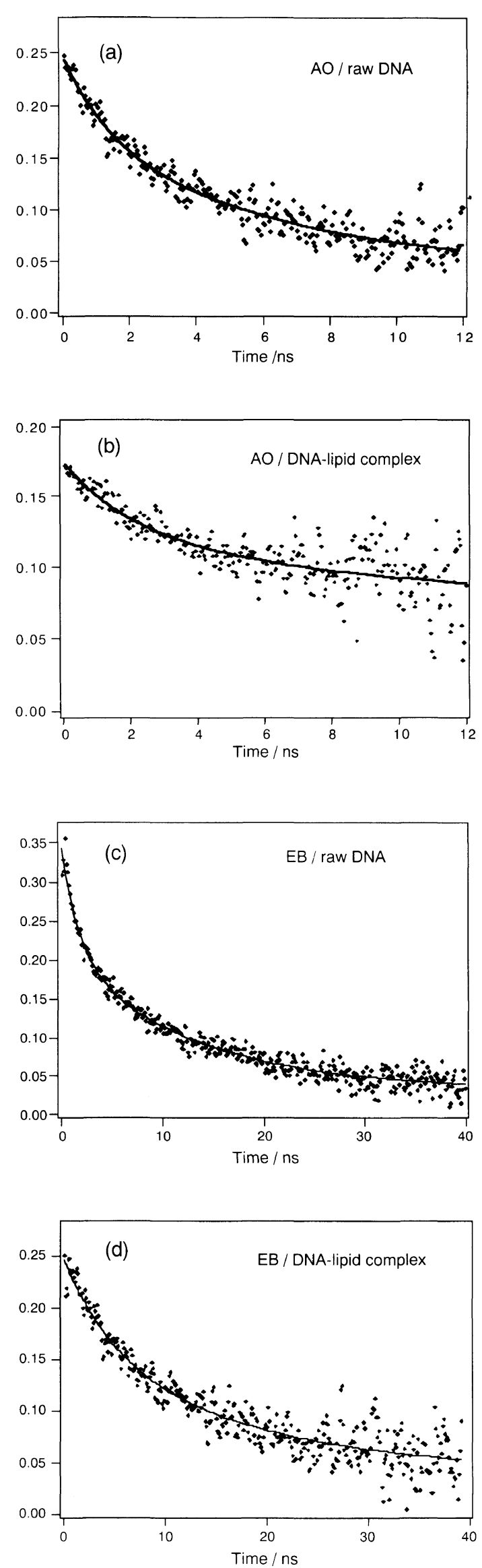

Figure 4. Time-resolved FPA decay profiles for (a) AO with the raw DNA in buffer, (b) AO with the DNA-lipid complex in $\mathrm{CHCl}_{3} /$ $\mathrm{MeOH} / \mathrm{H}_{2} \mathrm{O}$, (c) EB with the raw DNA in buffer, and (d) EB with the DNA-lipid complex in $\mathrm{CHCl}_{3} / \mathrm{MeOH} / \mathrm{H}_{2} \mathrm{O}$. The fitted curves are also shown. to the raw DNA or the DNA-lipid complex are presented in Figure 4. The data points of the decay for the DNA-lipid complex samples, in particular for $\mathrm{AO} /$ DNA-lipid complex, have larger distribution than those for the raw DNA samples. Even though we increased the integration counts, the distribution of the data was still large. This may reflect the heterogeneity of the local flexibility or structure of the DNA-lipid complex around the intercalation sites.

The decay curves were fitted with a double-exponential function,

$$
r(t)=A_{1} \exp \left(-t / \tau_{1}\right)+A_{2} \exp \left(-t / \tau_{2}\right)+A_{3}
$$

just because this function gives a satisfactory fit to the experimental data in the time range monitored. The fitted curves are also presented in Figure 4. According to the theoretical models treating DNA as a flexible rod, the twisting motions of DNA helix would lead to a nonexponential decay of the FPA which gives the torsional rigidity constant. ${ }^{4,9,10}$ In our case, however, the FPA decays could not be fitted sufficiently with a function of the non-exponential decay. The time constants and the relative contribution of each component of the FPA decays are listed in Table II. For all the samples, the two time constants for each FPA decay are several times different from each other and have similar pre-exponential factors. When we compare the time constants for the raw DNA samples and those for the DNA-lipid complex samples, both the longer and shorter components are about 2 times larger in the DNA-lipid complex. Thus the decays are slower in the DNA-lipid complex than those in the raw DNA. This result indicates that the twisting motion of the DNA-lipid complex is slower than that of the raw DNA, since the slower decay of FPA reflects the slower depolarizing motion. It should be because the long chains of lipid molecules attached around the DNA helix give some influences on the internal motions of the helix, and as a result, make the twisting motions slower. The viscosity of the $\mathrm{CHCl}_{3} /$ $\mathrm{MeOH} / \mathrm{H}_{2} \mathrm{O}$ solvent ( 0.62 cpoise) is about 2 times smaller than that of the aqueous buffer $(1.34$ cpoise $)$. The smaller viscosity of the $\mathrm{CHCl}_{3} / \mathrm{MeOH} / \mathrm{H}_{2} \mathrm{O}$ solvent is reflected by the steady-state FPA of the dyes without DNA helix (see Table I). Therefore, the lipid part restricts the twisting motion more strongly than the $\mathrm{CHCl}_{3} / \mathrm{MeOH} /$ $\mathrm{H}_{2} \mathrm{O}$ solvent reduces the viscosity.

We suppose that the twisting motion of the double helix is accompanied by the motion of the flexible chains of lipid molecules which are bound loosely or rigidly around the DNA helix by ionic interaction. It remains unknown here which part or what kind of property of the chain-like molecules attached to the helix plays a key role of controlling the twisting motions.

The two components of each FPA decay having similar population and significantly different values indicate that there are slower and quicker elements for twisting motions having similar contribution. It seems probable that DNA helix is such a long and flexible filament that each cylindrical rods are in different states, e.g., in linear and curved parts, or in intermediate and end parts of the filament. Therefore, such a difference would be reflected by the larger and smaller time constants showing slower and quicker motions of the DNA helix. The 
Table II. Relaxation parameters ${ }^{\mathrm{a}}$ for AO and EB intercalated in the raw DNA or the DNA-lipid complex

\begin{tabular}{cccccccc}
\hline Dye/DNA species & $A_{1}$ & $\tau_{1} / \mathrm{ns}$ & $A_{2}$ & $\tau_{2} / \mathrm{ns}$ & $A_{3}$ & $\tau_{\text {ave }}$ bs & $r(0)^{\mathrm{c}}$ \\
\hline AO/raw DNA & 0.014 & 6.8 & 0.095 & 1.4 & 0.037 & 4.6 & 0.27 \\
AO/DNA-lipid complex & 0.058 & 16.3 & 0.056 & 2.2 & 0.061 & 9.4 & 0.18 \\
EB/raw DNA & 0.18 & 12.7 & 0.14 & 1.6 & 0.031 & 7.9 & 0.35 \\
EB/DNA-lipid complex & 0.11 & 22.3 & 0.10 & 5.4 & 0.033 & 14.2 & 0.24 \\
\hline
\end{tabular}

${ }^{\mathrm{a}}$ Each decay was fitted by the function: $r(t)=A_{1} \exp \left(-t / \tau_{1}\right)+A_{2} \exp \left(-t / \tau_{2}\right)+A_{3} . \quad{ }^{\mathrm{b}} \tau_{\mathrm{ave}}=\left(A_{1} \tau_{1}+A_{2} \tau_{2}\right) /\left(A_{1}+A_{2}\right) . \quad{ }^{\mathrm{c}} r(0)=A_{1}+A_{2}+A_{3}$.

average time constants, $\tau_{\text {ave }}=A_{1} \tau_{1}+A_{2} \tau_{2}$, for both dyes are just twice as large as $\tau_{\text {ave }}$ for dye/raw DNA systems, irrespective of the kind of the dye molecules. The twisting motions of DNA double helix are supposed to be suppressed to an extent where the relaxation time constants become twice by the complex formation with the lipid molecules.

The existence of the time-independent component, $A_{3}$, means that the FPA relaxation has a component much longer than nanosecond timescale. This is probable because the twisting motion should have a certain limited amplitude angle. The values of the $A_{3}$ in Table II do not show marked dependence on the existence or absence of the lipid molecules or on the kinds of the dyes.

The values of FPA at $t=0, r(0)$, represent the extent of fluorescence depolarization which occurs faster than the time resolution of our apparatus $(\approx 1.5 \mathrm{~ns})$. The fast fluorescence depolarization is caused either by the difference in transition moment between the excitation and emission processes or by the fast rotational relaxation of the dye. In Table II the $r(0)$ values obtained as $A_{1}+A_{2}+A_{3}$ of the fitted parameters are also listed. For both dyes the DNA-lipid complex sample seems to show smaller $r(0)$ than the sample of the raw DNA. It is known that the dye intercalating to DNA shows rotational relaxation in hundred-picoseconds range resulting from the wobbling motion in the nucleic acid-base pair compartment. The smaller $r(0)$ for the DNA-lipid complex samples might be due to the lower viscosity of the solvent leading to a faster wobbling motion.

When we compare the relaxation constants for the two dyes, EB shows about twice as large values of the decay component as AO both for the raw DNA and the DNA-lipid complex samples. This fact may suggest that the twisting motion is affected by the structure or the size of the intercalating dye. This might be because the extent of the distortion of DNA helix induced by the intercalation depends on the structure or the size of the dye.

In conclusion, we have measured time-resolved FPA and other spectroscopic properties of the intercalating dyes to illustrate the effect of lipid molecules attached around DNA helix on twisting motions of the DNA helix. The twisting motions become slow by twice with the complex formation of the lipid molecules with the DNA helix. There seems to be at least two kinds of twisting motions, which are separated as slower and quicker ones.

Acknowledgment. This work is supported in part by a Grant-in-Aid for Scientific Research on Priority Areas (No. 277/08246211) from The Ministry of Education, Science, Sports and Culture of Japan.

\section{REFERENCES}

1. P. L. Felgner and G. M. Ringold, Nature, 337, 387 (1989).

2. T. C. Boles, J. H. White, and N. R. Cozzarelli, J. Mol. Biol., 213, 931 (1990).

3. S. B. Fujimoto, J. H. Shibata, R. L. Schurr, and J. M. Schurr, Biopolymers, 24, 1009 (1985)

4. S. A. Allison and J. M. Schurr, Chem. Phys., 41, 35 (1979).

5. Y. Okahata, K. Ijiro, and Y. Matsuzaki, Langmuir, 9, 19(1993).

6. K. Ijiro and Y. Okahata, J. Chem. Soc., Chem. Commun., 1339 (1992).

7. K. Tanaka and Y. Okahata, J. Am. Chem. Soc., 118, 10679 (1996).

8. T. Tao, Biopolymers, 8, 609 (1969).

9. M. D. Barkley and B. H. Zimm, J. Chem. Phys., 70, 2991 (1979).

10. J. M. Schurr, Chem. Phys., 84, 71 (1984). 